\title{
Correction to: Diet supplemented with phytochemical epigallocatechin gallate and probiotic Lactobacillus fermentum confers second generation synbiotic effects by modulating cellular immune responses and antioxidant capacity in aging mice
}

\author{
Rohit Sharma ${ }^{1}$ - Madhu Kumari $^{2} \cdot$ Amita Kumari $^{2} \cdot$ Anamika Sharma $^{1} \cdot$ Ashu Gulati $^{2} \cdot$ Mahesh Gupta $^{2}$. \\ Yogendra Padwad ${ }^{1}$
}

Published online: 28 June 2019

(c) Springer-Verlag GmbH Germany, part of Springer Nature 2019

\section{Correction to: European Journal of Nutrition https://doi.org/10.1007/s00394-018-01890-6}

Authors of the original article have observed an inadvertent error in their manuscript post-publication. The western blot images of $\beta$-tubulin protein bands used in Fig. 9c, $d$ are not correct. On re-examining, we realized that $\beta$-tubulin band images from another experiment were inadvertently selected, and this escaped our attention at that time. The actual images of the $\beta$-tubulin protein bands for Fig. 9c, $d$ are hereby attached, and the figure has been modified accordingly. This error does not alter or affect the result outcome and conclusions that have been reached in this paper. We sincerely apologize for this mistake and regret any inconvenience it may have caused.

Rohit Sharma

rohit25sharma@gmail.com

$\triangle$ Yogendra Padwad yogendra@ihbt.res.in

1 Pharmacology and Toxicology Laboratory, Food and Nutraceutical Division, CSIR-Institute of Himalayan Bioresource Technology, Palampur 176061, India

2 Food and Nutraceutical Division, CSIR-Institute of Himalayan Bioresource Technology, Palampur 176061, India 


\begin{tabular}{|c|c|c|c|c|c|}
\hline \multicolumn{4}{|c|}{ Original Figure } & \multicolumn{2}{|l|}{ Revised Figure } \\
\hline \multicolumn{3}{|c|}{$--\cdots+-\infty$} & Nrf-2 & $---\cdots--\infty$ & Nrf-2 \\
\hline 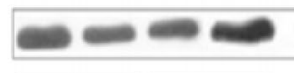 & $=0$ & $=0$ & $\beta$-Tubulin & & B-Tubulin \\
\hline Control & PRO & SYN & & $\overline{\text { Control }} \stackrel{\text { PRE }}{ }$ & \\
\hline$m=$ & $1+1$ & 14 & $\mathrm{NF}-\kappa \mathrm{B}$ & T & $\mathbf{N F - \kappa B}$ \\
\hline 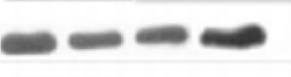 & $=0$ & $-\infty$ & $\beta$-Tubulin & - & $\beta$-Tubulin \\
\hline Control & PRO & SYN & & $\overline{\text { Control }} \overline{\text { PRE }} \overline{\text { PRO }}$ & \\
\hline
\end{tabular}

